\title{
20. Fortbildungsbildungskongress, Davos 2004
}

\section{Eine neue Volkskrankheit im Fokus}

\begin{abstract}
A llergien sind unbestritten heute eine „Volkskrankheit“. Dies war in den 70er Jahren noch nicht so. Wer damals von einer Zunahme der Allergien sprach, wurde als unkritisch oder Wichtigtuer betrachtet oder gar der „Hysterie“ bezichtigt. Dabei fiel bereits damals vielen Allergologen und Dermatologen in der Praxis auf, dass wesentlich mehr Kinder mit Neurodermitis oder junge Erwachsene mit Asthma oder Heuschnupfen Hilfe suchten. Es gab aber keine epidemiologischen Studien; diese begannen in Deutschland erst Ende der 80er Jahre, die erste in Düsseldorf im Rahmen des „Wirkungskatasters" der Landesregierung von Nordrhein-Westfalen, in die vom damaligen Medizinischen Institut für Umwelthygiene auch die Erfassung allergischer Phänomene und Krankheitsbilder mit eingeschlossen wurde. Kurz danach begannen in München - faktisch initiiert durch die politische Problematik der Wiederaufbereitungsanla-
\end{abstract}

\section{Wissenschaftliches Programm}

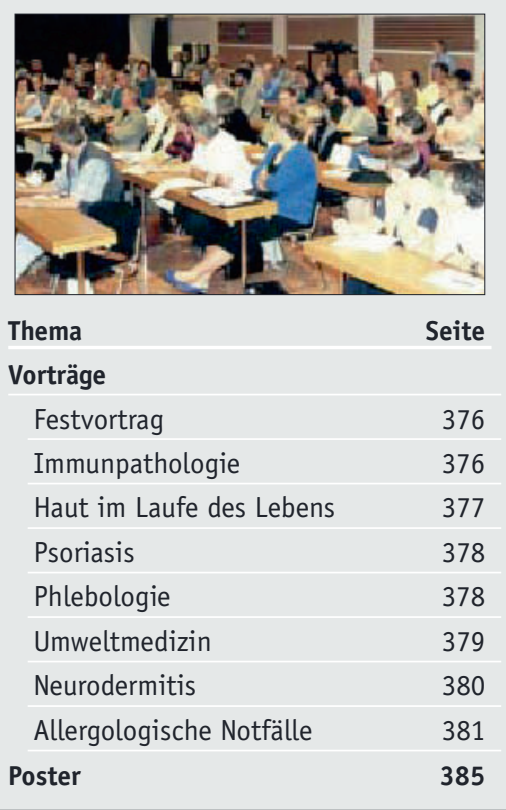

ge in Wackersdorf/Oberpfalz - erste epidemiologische Untersuchungen zur Häufigkeit von Allergien in verschiedenen Gegenden Bayerns. 1989 trafen sich die Gruppen und stellten fest, dass - entgegen vorgefasster Meinungen tatsächlich überraschend hohe Prävalenzen von allergischen Erkrankungen $\mathrm{zu}$ beobachten waren, und noch wesentlich höher war die Rate der Sensibilisierungen. Heute zweifelt niemand mehr daran; die Ursachen für diese $\mathrm{Zu}$ nahmen sind jedoch nach wie vor ungeklärt, trotz vieler und durchaus attraktiver Hypothesen (Urwaldhypothese, Hygienehypothese, Einfluss von Umweltschadstoffen etc.).

In diesem Zeitraum der historischen Entwicklung, der im Bereich der experimentellen Immunologie und Allergologie eine ungeheuere Wissensexplosion brachte, finden wir die Tradition der Davoser Kongresse zu den Fortschritten der Allergologie, Immunologie und Dermatologie, die von Prof. Dr. Dr. Siegfried Borelli 1979 mit dem ersten großen Kongress in Davos ins Leben gerufen wurde. Von Anfang an entsprach es dem Stil dieser Tagung, ausgewählte Themen aus dem gesamten Bereich der Allergologie und für alle an Allergologie interessierten Ärzte anzubieten, die in Form von Vorträgen, Symposien und Seminaren mit praktischen Übungen gestaltet werden. Neben den großen Vorträgen steht am Nachmittag die Arbeit in kleinen Gruppen mit praktischen Übungen zu vielfältigen Aspekten der Allergologie, Dermatologie und Immunologie.

In diesem Jahr sind besondere Schwerpunkte die Themen „Immundefekte“, „Umweltmedizin“, „Neurodermitis“, „Kinderdermatologie“, „Aknetherapie“ sowie „allergologische Notfälle“. Der Morgen beginnt mit einer Auswahl interessanter Kasuistiken in Form einer „Dia-Klinik“. Immer von Interesse sind auch die aktuellen
Kongressdaten auf einen Blick

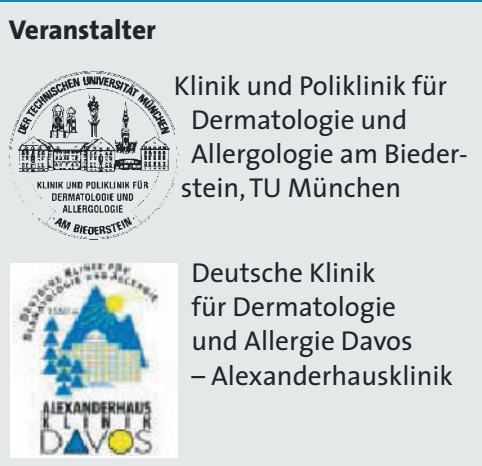

Wissenschaftliche Leitung
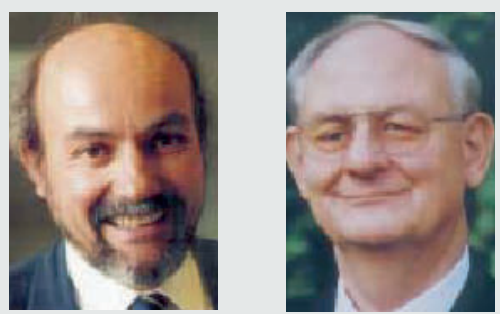

Univ.-Prof. Dr. Dr. Johannes Ring

Univ.-Prof. Dr. Dr. Siegfried Borelli

\section{Information}

Nora Enderlein

Tel.: (0o 49-89) 41 40-32 05

Fax: (0o 49-89) 41 40-31 73

E-Mail: Kongresse.derma@Irz.tum.de

Ergebnisse zu den Effekten der Klimatherapie auf Allergien und Hauterkrankungen in der geschützten Hochgebirgstallage von Davos. Auch gesundheitspolitische Themen werden berücksichtigt, dies ist besonders interessant in einem Jahr, in dem sich das Gesundheitssystem in Deutschland in wesentlichen Teilen von Grund auf ändert, ohne dass die Folgen derzeit bereits absehbar wären.

Wir freuen uns, Sie in Davos begrüßen zu dürfen, und wünschen Ihnen und allen Teilnehmern eine interessante und fruchtbringende Tagung.

Ihr

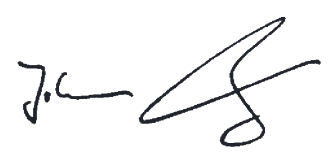

Prof. Dr. med. Dr. phil. Johannes Ring 